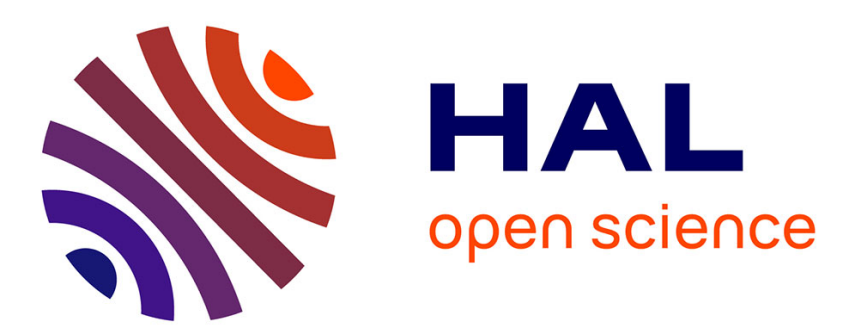

\title{
ÉTUDE DES PROPRIÉTÉS MAGNÉTIQUES DU NITRURE DE CHROME CrN
}

\author{
J. Chaussy, Robert Georges, P. Mollard, J. Voiron
}

\section{To cite this version:}

J. Chaussy, Robert Georges, P. Mollard, J. Voiron. ÉTUDE DES PROPRIÉTÉS MAGNÉTIQUES DU NITRURE DE CHROME CrN. Journal de Physique Colloques, 1971, 32 (C1), pp.C1-977-C1-979. 10.1051/jphyscol:19711347 . jpa-00214382

\section{HAL Id: jpa-00214382 https://hal.science/jpa-00214382}

Submitted on 1 Jan 1971

HAL is a multi-disciplinary open access archive for the deposit and dissemination of scientific research documents, whether they are published or not. The documents may come from teaching and research institutions in France or abroad, or from public or private research centers.
L'archive ouverte pluridisciplinaire HAL, est destinée au dépôt et à la diffusion de documents scientifiques de niveau recherche, publiés ou non, émanant des établissements d'enseignement et de recherche français ou étrangers, des laboratoires publics ou privés. 


\title{
ÉTUDE DES PROPRIÉTÉS MAGNÉTIQUES DU NITRURE DE CHROME CrN
}

\author{
J. CHAUSSY \\ Centre de Recherches sur les Très Basses Températures \\ R. GEORGES, P. MOLLARD et J. VOIRON \\ Laboratoire d'Electrostatique et de Physique du Métal \\ C. N. R. S., Cedex 166, Grenoble, France
}

\begin{abstract}
Résumé. - Nous discutons, au moyen du modèle de Bean et Rodbell, et sur la base de mesures nouvelles que nous décrivons (chaleur spécifique, magnétostriction spontanée à $0^{\circ} \mathrm{C}$ ) la transition magnétique du premier ordre observée dans CrN. On montre que cette transition peut être attribuée à un simple mécanisme de magnétostriction spontanée.

Abstract. - The first order magnetic transition observed in $\mathrm{CrN}$ is analyzed, with the model proposed by Bean and Rodbell, on the basis of new results that we report (specific heat, spontaneous magnetization at $0^{\circ} \mathrm{C}$ ). It is shown that the transition cannot be interpreted as only induced by magnetostriction.
\end{abstract}

Introduction. - Le nitrure de chrome CrN présente à la température ambiante la structure cubique à faces centrées du chlorure de sodium [1,2]. Lorsque la température diminue, la maille se déforme brusquement au voisinage de $0^{\circ} \mathrm{C}$, la phase basse température présentant des écarts importants à la symétrie c. f. c. $\mathrm{Au}$ cours de cette transition du premier ordre, les moments des ions chrome s'ordonnent suivant un arrangement antiferromagnétique de quatrième espèce $[1,2]$. L'existence d'une transition magnétique du premier ordre, le maintient jusqu'à une température élevée d'un arrangement généralement instable, et l'amplitude exceptionnelle de certaines déformations du réseau constituent autant d'anomalies auxquelles il faut sans doute apporter une explication commune.

Le modèle de Bean et Rodbell [3] est souvent invoqué pour rendre compte des transitions du premier ordre entre une phase ordonnée et une phase paramagnétique. Dans cette communication, nous discutons de son application au nitrure de chrome.

Situation expérimentale. - Les mesures de susceptibilité paramagnétique [4] conduisent à une température de Curie de $-2600^{\circ} \mathrm{K}$ et un moment effectif de $4,08 \mu_{\mathrm{B}}$ en accord avec le spin $\frac{3}{2}$ de l'ion $\mathrm{Cr}^{+++}$. Cependant, les mesures par diffraction des neutrons dans la phase ordonnée [1,2] font apparaître un moment de $2,4 \mu_{\mathrm{B}}$, ce qui correspond à un facteur gyromagnétique de 1,6. L'écart entre ces deux résultats pourrait provenir de la variation thermique des interactions d'échange. En effet, si l'on admet qu'elles varient linéairement avec la température dans le domaine paramagnétique, $\theta_{\mathrm{p}}$ se met sous la forme [5]

$$
\theta_{\mathrm{p}}=\theta_{\mathrm{p}}^{\mathrm{o}}(1+\gamma T) \text {. }
$$

Cette variation permet de retrouver la constante de Curie correspondant au moment observé dans la phase ordonnée si l'on admet $\gamma=-3,4 \times 10^{-4}\left({ }^{\circ} \mathrm{K}\right)^{-1}$, ce qui correspond à $\theta_{\mathrm{p}}^{0}=-1650^{\circ} \mathrm{K}$. Dans l'approximation du champ moléculaire les interactions d'échange $J_{1}$ et $J_{2}$ entre premiers et seconds voisins peuvent être déduites de $\theta_{\mathrm{p}}^{0}$ et de la température de transition $\theta_{\mathrm{c}}$ considérée comme la température de Néel de l'arrange- ment de quatrième espèce. On obtient ainsi, dans la phase non distordue $J_{1} \simeq 25 \mathrm{oK}^{\mathrm{K}}$ et $J_{2} \simeq 50 \mathrm{\circ}$, les interactions d'échange étant définies par l'hamiltonien

$$
\mathscr{H}=-2 \sum_{i, n} J_{1} \mathbf{S}_{\mathrm{i}} \mathbf{S}_{\mathrm{i}+\mathrm{n}}-2 \sum_{i, m} J_{2} \mathbf{S}_{\mathrm{i}} \mathbf{S}_{\mathrm{i}+\mathrm{m}} .
$$

A la transition la susceptibilité magnétique subit une discontinuité [4] qu'on peut attribuer à une augmentation de $70 \%$, dans la phase ordonnée, des interactions d'échange entre sous-réseaux de spins opposés.

L'application d'une pression hydrostatique fait croître $\theta_{\mathrm{c}}$ de $0,9 \circ \mathrm{K}$ par kilobar.

L'étude cristallographique [2] met en évidence, à la transition, une anomalie de volume d'environ $-0,6 \%$; une déformation orthorhombique apparaît dans la phase ordonnée, et les quantités $\varepsilon, \eta$ et $\xi$ qui mesurent les écarts relatifs des paramètres de la maille par rapport à la symétrie cubique (Fig. 1) sont à $22^{\circ} \mathrm{K}$,

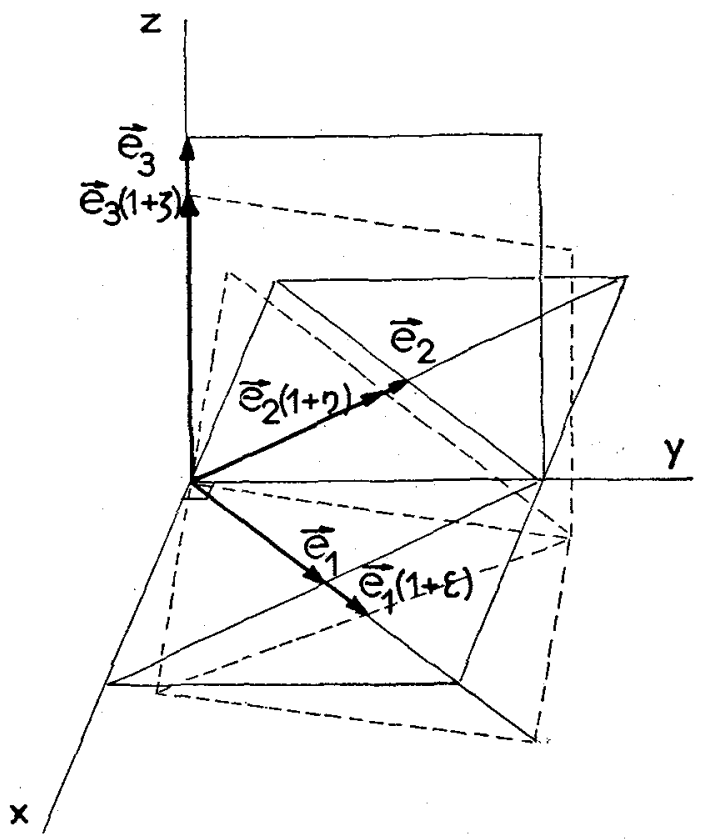

FIG. 1. - Déformations orthorhombiques de la maille de $\mathrm{CrN}$ à basse température. 
$\varepsilon=-1,48 \%, \eta=1,56 \%, \xi=-0,08 \%$ [2]. Nous avons mesuré ces quantités immédiatement au-dessous de la transition : les valeurs obtenues sont inférieures de 10 à $20 \%$ aux précédentes. Si cette variation est due à la magnétostriction spontanée, elle correspond à une diminution de l'aimantation des sous-réseaux de 5 à $10 \%$ entre le zéro absolu et la transition. La déformation la plus importante est constituée par le changement des distances entre plans ferromagnétiques premiers voisins [2]. Ces plans sont équidistants dans la structure c. f. c., mais dans la phase ordonnée, leur espacement augmente ou diminue de $4 \%$ selon que les plans consécutifs portent des spins de même sens ou de sens opposés (Fig. 2). Afin d'obtenir des informations sur

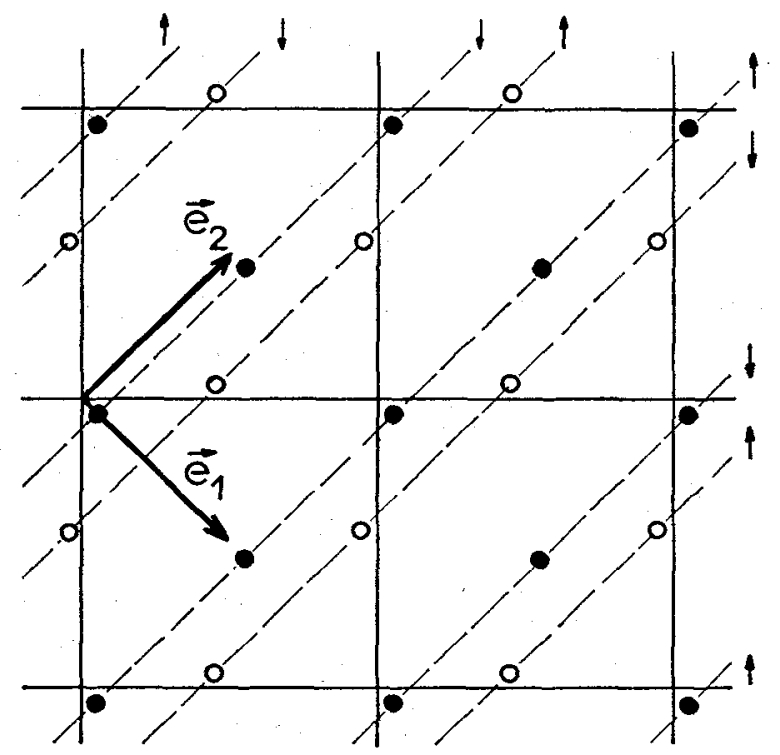

- Site de cote 0

O Site de cote $1 / 2$

FIG, 2. - Modification des distances entre plans ferromagnétiques.

son comportement thermodynamique dans la phase ordonnée et à la transition, nous avons déterminé la chaleur spécifique du nitrure de chrome entre $20 \% \mathrm{~K}$ et $315 \mathrm{oK}$. Les mesures ont été effectuées sur un échantillon dépourvu d'oxygène (Fig. 3). La transition se manifeste, en températures croissantes, par un pic de chaleur spécifique à $8,5^{\circ} \mathrm{C}$. La température de la transition est très sensible à la composition chimique ; des écarts à la stœchiométrie, sans doute par défaut d'azote, sont à l'origine de l'élargissement de la région de transition. La chaleur latente déterminée à partir de l'aire de l'anomalie est de $29 \mathrm{~J} / \mathrm{g}$. Elle est en bon accord avec $28 \mathrm{~J} / \mathrm{g}$, valeur que l'on déduit de l'anomalie de volume et de l'effet de la pression $\operatorname{sur} \theta_{c}$, en utilisant la formule de Clapeyron $[2,3]$.

Discussion. - Selon le modèle de Bean et Rodbell [3], une transition du premier ordre peut résulter d'une variation rapide de l'énergie magnétique avec les distances interatomiques. On ne peut appliquer

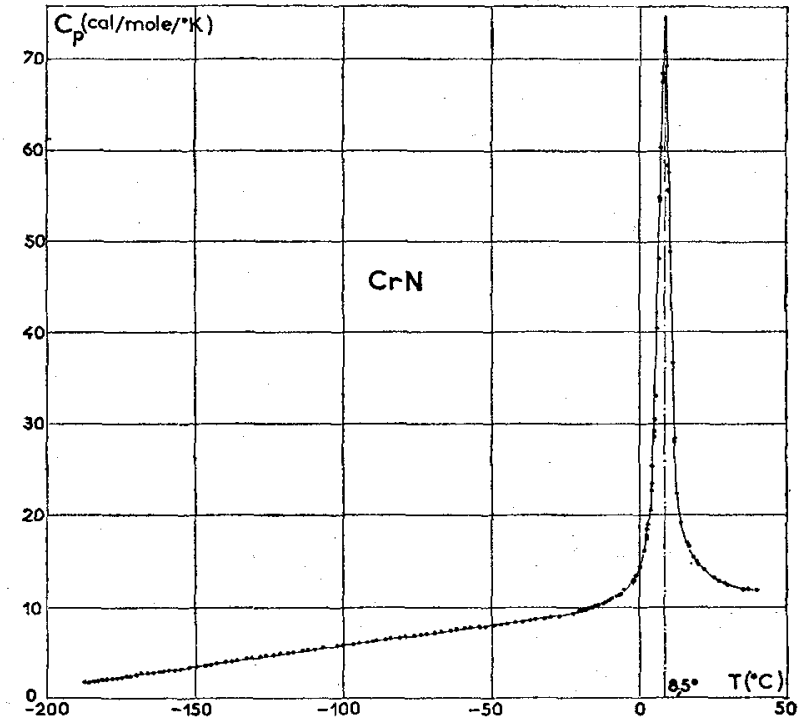

Fig. 3. - Chaleur spécifique de CrN.

directement ce modèle, les valeurs des coefficients élastiques du nitrure de chrome étant inconnues. Certaines remarques permettent cependant d'affirmer que ce mécanisme n'est pas à l'origine de la transition du premier ordre observée.

1. En introduisant les coefficients élastiques $c_{11}, c_{12}$ et $c_{44}$ et les paramètres $j_{1}=d \log J_{1} / d \log r_{1}$ et $j_{2}=d \log J_{2} / d \log r_{2}$ qui caractérisent les variations des interactions d'échange avec les distances interatomiques, l'anomalie de volume et l'anomalie du paramètre $c$, au zéro absolu sont données par :

$$
\begin{aligned}
& \left(\frac{\mathrm{d} V}{V}\right)_{0}=\frac{\vec{N} S^{2} j_{2} J_{2}}{c_{11}+2 c_{12}} \\
& \left(\frac{\mathrm{d} C}{C}\right)_{0}=\frac{\bar{N} S^{2} j_{2} J_{2}}{c_{11}+2 c_{12}} \cdot \frac{c_{11}+3 c_{12}}{\left(c_{12}-c_{11}\right)} .
\end{aligned}
$$

Elles sont toutes deux négatives, ce qui conduit à l'inégalité $c_{12}>c_{11}$; or une telle inégalité n'est jamais respectée dans des composés voisins de $\mathrm{CrN}$.

2. L'égalité des énergies librès à la transition est confirmée par le bon accord entre la valeur expérimentale de la chaleur latente et celle que l'on calcule par la relation de Clapeyron. Dans cette hypothèse, en raison de la magnétostriction spontanée, la température de Néel varie comme le carré de l'aimantation relative des sous-réseaux

$$
\theta_{\mathrm{N}}(\sigma)=\theta_{\mathrm{N}}(0)\left(1+\alpha \sigma^{2}\right) \text {. }
$$

Pour assurer l'égalité des énergies libres entre la phase paramagnétique et la phase ordonnée présentant une aimantation relative égale à 0,9 , on est conduit à attribuer à $\alpha$ une valeur voisine de 2,3. Par ailleurs les arrangements antiferromagnétiques d'ordre (I, II, ou III) qui sont plus stables que l'arrangement d'ordre IV en l'absence de magnétostriction [6], ont nécessairement des températures de Néel inférieures à $280^{\circ} \mathrm{K}$, sinon elles apparaîtraient à partir de la phase paramagnétique. Cette remarque conduit à attribuer à $\alpha$ 
une valeur au moins égale à 3,4. L'incompatibilité entre ces deux conditions et les très fortes valeurs de $\alpha$ qu'elles impliquent toutes deux indiquent que la transition ne peut être due uniquement à des interactions magnétoélastiques.

Conclusion. - Bien que disposant de peu d'informations sur la valeur des coefficients élastiques et magnétoélastiques, nous avons pu montrer qu'un phénomène purement magnétoélastique était difficilement compatible avec l'établissement d'un arrangement antiferromagnétique de quatrième espèce et avec la nature des déformations observées, bien qu'il faille améliorer le modèle en tenant compte des variations des coefficients élastiques, en raison de l'importance des déformations.

\section{Bibliographie}

[1] Corliss (L. M.), Elliott (N.) et Hastings (J. M.), Phys. Rev., 1960, 117, 929

[2] Bertaut (E. F.), Eddine (E. N.) et Sayetat (F.) C. R. Acad. Sci. Paris, 1969, 269B, 574.

[3] Bean (C. P.) et Rodbell (D. S.), Phys. Rev., 1962 126, 104
[4] Bloch (D.), Mollard (P.) et Voiron (J.), C. R. Acad. Sci. Paris, 1969, 269B, 553

[5] Néel (L.), Ann. Phys., 1937, 8, 327.

[6] SMART (J. M.), Magnetism III, 1963 (Rado et Suhl Edt.). 